\title{
AVALIAÇÃO IN VITRO DO EXTRATO DA Carapa guianensis (MELIACEAE) E DA Uncaria guianensis (RUBIACEAE) SOBRE Malassezia pachydermatis ISOLADA DE PRIMATAS NÃO HUMANOS NO ESTADO DO ACRE
}

Alexandre Augusto da Rocha ${ }^{1}$, Tamyres Izarelly Barbosa da Silva ${ }^{2}$, Sandra Albuquerque Lima Ribeiro ${ }^{3,}$ Alex Cicinato Paulino de Oliveira ${ }^{4}$, Sara Lucena de Amorim ${ }^{4^{*}}$.

${ }^{1}$ Médico veterinário, Universidade Federal do Acre - UFAC, BR-364, Rio Branco - AC, 69920-900

2 Docente, Laboratório de Doenças Infecciosas dos Animais da Universidade Federal do Acre - UFAC, BR-364, Rio Branco - AC, 69920-900 ${ }^{3}$ Docente, Laboratório de Fitopatologia da Universidade Federal do Acre - UFAC, BR-364, Rio Branco - AC, 69920-900

${ }^{4}$ Docentes do curso de Medicina Veterinária da Universidade Federal de Rondônia UNIR, Rolim de Moura - RO, CEP 76.940,000 (cicinato@bol.com.br, saravet.la@bol.com.br

Recebido em: 15/08/2020 - Aprovado em: 15/09/2020 - Publicado em: 30/09/2020 DOI: 10.18677/EnciBio 2020C12

\begin{abstract}
RESUMO
A malasseziose é uma doença fúngica desencadeada por um agente oportunista presente na microbiota cutânea dos animais domésticos e silvestres. Sabendo que os antifúngicos convencionais nem sempre apresentam resultados satisfatórios e que esta enfermidade pode causar sérios riscos à conservação da fauna acreana, se faz necessário o uso de métodos alternativos e naturais, os quais tenham a capacidade de combater a infecção com menos reações adversas possíveis. Sendo assim, o objetivo deste trabalho foi analisar a ação antifúngica da Carapa guianensis e da Uncaria guianensis, conhecidas popularmente como andiroba e unha de gato respectivamente, sobre o isolado de Malassezia pachydermatis de primatas não humanos do estado do Acre. O material vegetal utilizado foi raiz, caule e folha das plantas, sendo os extratos etanólicos produzidos na fundação de apoio à pesquisa do estado do Acre - FUNTAC. Amostras cutâneas foram coletadas de primatas no Centro de Triagem de Animais Silvestres por avulsão de pêlos, onde foi realizado o isolamento da Malassezia pachydermatis no laboratório de Fitossanidade da Universidade Federal do Acre UFAC. As técnicas que foram utilizadas foram o método de disco difusão em ágar, diluição em ágar e difusão por poço. Observou-se que as amostras de ambos os extratos da Carapa guianensis e Uncaria guianensis não apresentaram atividade antifúngica frente a Malassezia pachydermatis na concentração de $100 \mathrm{mg} / \mathrm{mL}$ Acreditase que este resultado pode estar associado ao fator dose/concentração e/ou aos princípios ativos, sendo necessárias mais pesquisas para elucidar uma resposta satisfatória.
\end{abstract}

PALAVRAS CHAVE: andiroba; fitoterápico; fungos; macacos; unha de gato. 


\title{
IN VITRO EVALUATION OF THE EXTRACT OF THE Carapa guianensis (MELIACEAE) AND THE Uncaria guianensis (RUBIACEAE) ABOUT Malassezia pachydermatis ISOLATED FROM PRIMATES IN THE STATE OF ACRE
}

\begin{abstract}
Malasseziosis is a fungal disease, which the opportunistic agent is present in the cutaneous microbiota of domestic and wild animals. Knowing that the use of conventional medicines based on antifungal drugs is not always guaranteed success and that this disease can cause serious risks to the conservation of the Acrean fauna, it is necessary to use alternative and natural methods, which have the capacity to combat the infection with fewer possible adverse reactions. Thus, the objective of this work was to analyze the antifungal action of Carapa guianensis and Uncaria guianensis, popularly known as andiroba and cat claw, respectively, on the isolate of Malassezia pachydermatis of primates from the state of Acre. The plant material used was root, stem and leaf of the plants, being the ethanolic extracts produced in the Foundation of Technology of the State of Acre (FUNTAC). Cutaneous samples were collected from primates at the Screening Center for Wild Animals (CETAS) by avulsion of hairs, where the isolation of Malassezia pachydermatis was carried out in the phytosanitary laboratory of the Federal University of Acre (UFAC). The techniques that were used were the disk diffusion method in ágar, ágar dilution and diffusion technique per well. The results obtained in this work were that the samples of both extracts of Carapa guianensis and Uncaria guianensis did not present antifungal activity against Malassezia pachydermatis at the concentration of $100 \mathrm{mg} / \mathrm{ml}$. This result may be associated with the dose / concentration factor and / or active principles, thus further research is needed to elucidate a satisfactory response.
\end{abstract}

KEYWORDS: andiroba; herbal remedy; fungal disease; cat's claw.

\section{INTRODUÇÃO}

O gênero Malassezia faz parte de um complexo de leveduras de caráter lipofílico, os quais se encontram presentes na superfície cutânea de aves e mamíferos, possuindo 13 espécies identificadas: $M$. pachydermatis, $M$. sympodialis, $M$. restrica, $M$. globosa, $M$. slooffiae, $M$. obtusa, $M$. furfur, $M$. japonica, $M$. yamatoensis, $M$. nana, $M$. caprae e M. equina (LÓPEZ, 2008; CABAÑES et al., 2011; MELCHERT, 2011). Este fungo apresenta-se sob a forma elipsoide, esférica ou alongada e se reproduz por brotamento unipolar (TRABULSI, 2008).

A $M$. pachydermatis é uma das espécies mais importantes presente na microbiota normal dos animais domésticos e silvestres, sendo patogênica apenas na ocorrência de alterações no sistema imune do hospedeiro ou na superfície de sua pele, propiciando a multiplicação da população fúngica cutânea (SANDOVAL, 2014). De todas as espécies do gênero, a $M$. pachydermatis, apesar de lipofílica, é a única não lipo-dependente, não necessitando de um ambiente rico em lipídios para seu crescimento (GUILLOT et al., 1998; ALBANO, 2009).

O tratamento da malasseziose é realizado com uso de antifúngicos tópicos e/ou sistêmicos (ILKIT et al., 2015). Uma das problemáticas da terapêutica é que esses fungos vêm desenvolvendo resistência à terapia antifúngica convencional, o que ocasiona uma suscetibilidade muito variável in vitro e in vivo, que consequentemente 
causa aumento progressivo do número de recidivas, acarretando a não responsividade aos fármacos antimicóticos usuais (REZENDE et al., 2008).

Sabendo que o uso de medicamentos convencionais nem sempre tem o sucesso garantido, se faz necessário o uso de métodos alternativos, os quais garantam o máximo de conforto ao animal e o mínimo de dano possível ao mesmo (FOY ; TREPANIER, 2010). Uma alternativa que vem sendo estudada desde a década de 90 é a utilização de fitoterápicos, os quais apresentam um custo baixo e, ao mesmo tempo causam poucos ou quase nenhum dano aos pacientes (HEIMERDINGER et al., 2006). Dentre as várias plantas já utilizadas com caráter fitoterápico, duas espécies se destacam pela sua variedade de utilidades: a espécie Carapa guianense conhecida popularmente como "andiroba", e a Uncaria guianensis, a "unha de gato" (FERRAZ et al., 2002).

A Carapa guianensis pertence à família Meliaceae e se distribui por toda a América do Sul, América Central, África tropical e Antilhas, sendo que no Brasil ela cresce em toda Bacia Amazônica, onde é encontrada com facilidade perto de áreas alagáveis próximas aos rios (NEVES et al., 2004). É considerada uma das espécies mais valiosas da Floresta Amazônica, sendo a madeira e o óleo extraído de suas sementes os produtos mais importantes com propriedades anti-inflamatórias (HENRIQUE;PENIDO 2014), antibacterianas (SILVA et al., 2015) e principalmente, como repelente de insetos, acaricidas e anti-helmíntica ( FARIAS et al., 2007, KLAUCK et al., 2015, AMORIM, 2016).

A Uncaria guianensis, pertence à família Rubiaceae, está distribuída por quase toda a América do Sul, tendo sua base de crescimento mais forte na região norte do Brasil, sendo que há registros de até 2 mil anos de utilização da mesma por tribos indígenas da região acreana e do Peru (MIRANDA et al., 2003, POLLITO, 2004). É uma planta utilizada para várias enfermidades, porém existem relatos do seu uso contra problemas de úlceras gástricas, alergias e até mesmo contra alguns fungos (REINHARD, 1999; SOJAKOVA et al., 2004; HEITZMAN et al., 2005).

Visto que existem poucas pesquisas relacionadas à atividade fungicida dessas plantas e que possuem diversas formas de uso, é necessária a realização de uma investigação mais profunda das mesmas, visando buscar um tratamento inovador para a malasseziose. Sendo assim, o objetivo deste trabalho foi analisar o extrato bruto etanólico das folhas, raiz e caule da Carapa guianensis e Uncaria guianensis sobre culturas isoladas de Malassezia pachydermatis (in vitro) de primatas através dos métodos de difusão em poços, diluição em ágar e disco-difusão em ágar.

\section{MATERIAL E MÉTODOS}

O experimento foi desenvolvido no Laboratório de Fitossanidade, no Laboratório de Doenças Infecciosas dos Animais na Universidade Federal do Acre (UFAC) e no Laboratório de Produtos Naturais da Fundação de Tecnologia de Pesquisa do estado do Acre (FUNTAC).

A coleta do material botânico foi realizada baseando-se nas metodologias de Cartaxo et al., (2010). As plantas foram coletadas no Parque Zoobotânico do Campus da UFAC de Rio Branco (958'29" sul e a 67웅'36" oeste), sendo identificadas e depositadas no herbário desta instituição, com exsicata de número 6421 (Carapa guianensis) e 6422 (Uncaria guianensis). A coleta da planta foi realizada no primeiro semestre do ano de 2015. 
Os materiais vegetais da raiz, folha e caule da Carapa guianensis e Uncaria guianensis foram coletados e submetidos à secagem ao ar livre por 48 horas, em seguida levados à estufa de ventilação forçada a $60^{\circ} \mathrm{C}$ por 24 horas, logo após, pesados e moídos. O preparo dos extratos etanólicos da folha, raiz e caule foram submetidas à extração através de uma solução hidroalcóolica a $70 \%$. Após sete dias de percolação, o material vegetal foi filtrado e submetido ao rota evaporador obtendo um material viscoso. Para uma eficiente evaporação do solvente, os materiais foram colocados em frascos de vidro tarados e em banho Maria, em seguida foram submetidos ao processo de liofilização a uma temperatura de aproximadamente $-30^{\circ} \mathrm{C}$ por 24 horas (MATOS, 2009).

A M. pachydermatis foi isolada de amostras cutâneas, colhidas por avulsão pilosa e raspado cutâneo superficial de primatas não humanos do Centro de Triagem de Animais Silvestres - CETAS, no estado do Acre. A coleta do material biológico foi aprovada pelo Sistema de Autorização e Informação em Biodiversidade - SISBIO, sob o número de protocolo 58122/2017.

O material biológico foi semeado em ágar Sabouraud Dextrose (ASD) e incubado de forma aeróbia à temperatura de $30^{\circ} \mathrm{C}$ em câmara incubadora do tipo BOD (Biochemical Oxygen Demand), sendo observado o crescimento de colônias características, por meio de análises macroscópicas, microscópicas e bioquímicas (NOGUEIRA et al., 2013).

Para a análise da atividade antifúngica do extrato das plantas, foram utilizados os métodos de diluição em ágar, difusão em poços e disco-difusão em ágar. A dose e concentração utilizadas nesse trabalho foi de $100 \mathrm{mg} / \mathrm{mL}$ em todas as técnicas. Os testes foram realizados em duplicatas.

$\mathrm{Na}$ difusão em poços, foram perfurados quatro poços de $12 \mathrm{~mm}$ de diâmetro em seis placas de ASD. Em cada poço foram distribuídos $20 \mathrm{~L}$ do extrato da raiz, caule, folha e no quarto poço apenas água destilada estéril, servindo como controle positivo. Em seguida, alçadas de colônias purificadas de $M$. pachydermatis foram semeadas uniformemente ao redor dos poços, deixado a placa em incubação à temperatura de $30^{\circ} \mathrm{C}$ em BOD, por até cinco dias (VITAL et al., 2003)

No método de disco difusão em ágar, discos de filtro de papel estéreis (Figura 1) foram submergidos nos extratos e distribuídos em seis placas, onde posteriormente, a $M$. pachydermatis foi semeada. As placas foram incubadas à temperatura de $30^{\circ} \mathrm{C}$ em BOD, por até cinco dias (NCCLS, 2003).

Para a técnica de diluição em ágar, os extratos de folha, caule e raiz foram diluídos em água destilada e inseridos na preparação do ASD, antes e após o processo de esterilização em autoclave (PINTO, 2003). Assim, foram preparadas 14 placas de Petri, as quais seis continham meio ASD com extratos adicionados de forma prévia à esterilização; seis placas com meio ASD com extratos adicionados após a esterilização; uma placa com meio ASD acrescido de antifúngico (cetoconazol para controle negativo); uma placa com meio ASD apenas, sendo o controle positivo.

Após a preparação destes meios, a $M$. pachydermatis foi semeada uniformemente sobre a superfície do ágar. Todas as placas foram incubadas à temperatura de $30^{\circ} \mathrm{C}$ em BOD, por até cinco dias. Os testes foram realizados em triplicata. Foi considerado como resultado positivo qualquer meio de cultivo cujo extrato criasse um halo de inibição sobre a $M$. pachydermatis, e considerado como negativo as placas que sofressem crescimento fúngico na extensão sobre os extratos utilizados. 


\section{RESULTADOS}

$\mathrm{Na}$ técnica de difusão em poços (Fig. 1A-B), observou-se crescimento de $M$. pachydermatis evidente em todos os poços contendo ou não (controle positivo) e os extratos da Carapa guianensis e da Uncaria guianensis, respectivamente.

FIGURA 1 - Distribuição de extratos vegetais em ágar Sabouraud Dextrose (ASD) para avaliação de atividade antifúngica in vitro frente a isolados de Malassezia pachydermatis. A) Extrato do caule da Carapa guianensis em poços no ASD; B) Extrato do caule da Uncaria guianensis em poços no ASD; C) Extrato da raiz, caule e folha da Carapa guianensis em discos no ASD; D) Extrato da raiz da Carapa guianensis diluído no ASD.

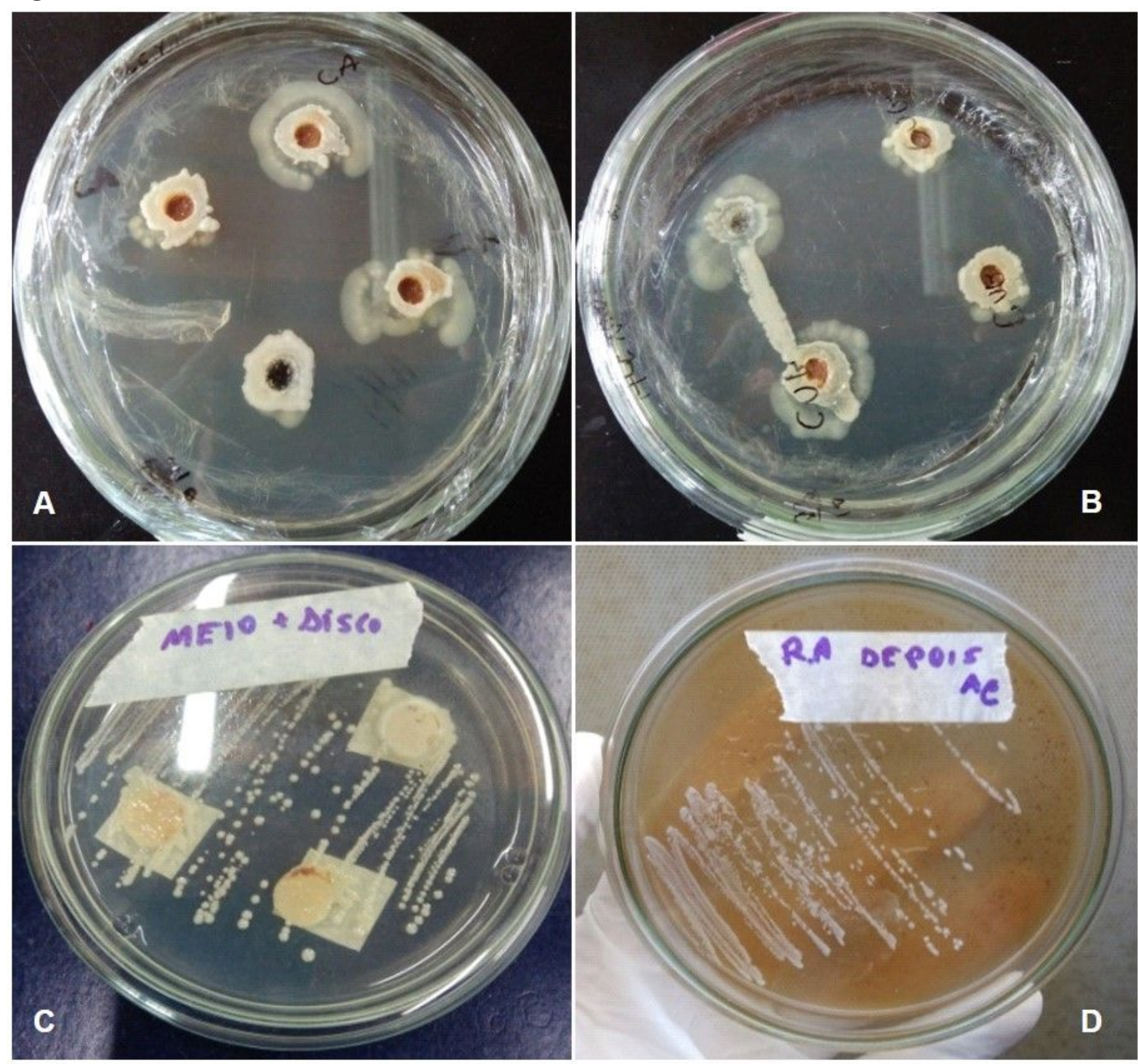

Pela técnica de disco-difusão, também foi observado o crescimento fúngico sobre os extratos testados, como demonstrado na Figura 1C. Na técnica de diluição em ágar, o crescimento da $M$. pachydermatis foi evidenciado em todas as placas contendo os extratos tanto da Carapa guianensis quanto da Uncaria guianensis (Fig. 1D). Na Tabela 1, é mostrado a atividade dos extratos perante todas as técnicas utilizadas. 
TABELA 1. Demonstração da atividade fungicida dos extratos etanólicos da Carapa guianensis e Uncaria guianensis sobre a Malassezia pachydermatis de primatas não humanos nas diferentes técnicas de diagnóstico estudadas.

\begin{tabular}{|c|c|c|c|c|c|}
\hline Extratos & Dose/Concentração & $\begin{array}{l}\text { Técnica } \\
\text { de } \\
\text { Difusão } \\
\text { em } \\
\text { poços }\end{array}$ & $\begin{array}{l}\text { Técnica } \\
\text { de } \\
\text { Disco- } \\
\text { Difusão } \\
\text { em } \\
\text { Ágar }\end{array}$ & $\begin{array}{l}\text { Técnica } \\
\text { de } \\
\text { Diluição } \\
\text { em Âgar }\end{array}$ & $\begin{array}{l}\text { Crescimento } \\
\text { fúngico }\end{array}$ \\
\hline${ }^{\star} \mathrm{R} . \mathrm{A}$ & $100 \mathrm{mg} / \mathrm{mL}$ & ${ }^{*}+$ & + & + & Positivo \\
\hline${ }^{*} \mathrm{C} . \mathrm{A}$ & $100 \mathrm{mg} / \mathrm{mL}$ & + & + & + & Positivo \\
\hline${ }^{\star} \mathrm{F} . \mathrm{A}$ & $100 \mathrm{mg} / \mathrm{mL}$ & + & + & + & Positivo \\
\hline${ }^{\star}$ R.U.G & $100 \mathrm{mg} / \mathrm{mL}$ & + & ${ }^{*}-$ & - & Positivo \\
\hline${ }^{*}$ C.U.G & $100 \mathrm{mg} / \mathrm{mL}$ & + & - & - & Positivo \\
\hline${ }^{*}$ F.U.G & $100 \mathrm{mg} / \mathrm{mL}$ & + & - & - & Positivo \\
\hline
\end{tabular}

\section{DISCUSSÃO}

Embora a $M$. pachydermatis tenha uma sensibilidade considerável a extratos e outros produtos naturais (CARDOSO et al., 2010), o resultado da pesquisa não se mostrou eficaz, supondo que fatores como dose/concentração, composição química dos extratos, época de coleta do ano e técnicas realizadas possam ter influenciado o resultado final do experimento.

Outros fatores também podem ter influenciado diretamente na condição do crescimento do fungo mediante os extratos. Antes mesmo da sua utilização nas placas, o processo de extração, o qual geralmente utilizado é o de destilação de vapor de água, em sua maioria gera algumas substâncias que podem acabar influenciando no experimento em si (SIMÕES et al., 2017). Outra condição que pode ter comprometido no resultado é que trabalhamos com extrato liofilizado e isso dificultou seu processo de diluição, o que justifica não termos utilizado concentrações mais altas.

Nascimento et al. (2013) afirmam que é necessário ficar atento também a dosagem e concentração do extrato que será utilizado, visto que usou concentrações baixas e obteve resultados positivos com o extrato de eucalipto sobre o fungo Thielaviopsis paradoxa, neste estudo a mesma não conseguiu repetir o processo com o extrato da Carapa guianensis, obtendo um efeito até menor que o próprio controle positivo.

Segundo Machado et al. (2013) a Carapa guianensis não surtiu efeito antifúngico sobre o Colletotrichum gloesporioides, sendo utilizado na concentração de 
$750 \mathrm{mg} / \mathrm{L}$, o que para os outros extratos usados na pesquisa, foram bem sucedidos, porém seu efeito foi bem próximo ao desejado, implicando que uma concentração mais elevada possa originar um melhor efeito fungicida, e em comparação, à dose utilizada no presente trabalho foi bem mais baixa $(100 \mathrm{mg} / \mathrm{mL})$, o que pode ter sido um fator determinante para a atividade negativa perante ao fungo.

Muitos estudos afirmam que boa parte dos óleos e extratos que são usados nas pesquisas possuem atividades terapêuticas muito eficientes. Porém, devido ao fato de existirem diferentes métodos e avaliações, comparar um extrato com outro acaba ficando cada vez mais difícil. Segundo a pesquisa feita por Amorim (2016), tanto o caule quanto a raiz da Carapa guianensis e Uncaria guianensis possuem reação positiva aos seguintes constituintes químicos: Fenóis, taninos, saponinas, esteróides, triterpenóides, antraquinonas, açucares redutores, ácidos orgânicos e flavonóides. De acordo com Castejon (2011) os fenóis e taninos presentes na maioria das plantas são compostos que possuem características fungicidas, porém em baixa quantidade, o que pode ter sido outro fator relevante na baixa atividade da Carapa guianensis e da Uncaria guianensis, já que essas plantas são consideradas por apresentarem grande quantidade de compostos fenólicos.

Existem fungos epifíticos, os quais convivem em simbiose com as plantas, e produzem substâncias como os flavonóides, que possuem certa atividade fungicida. Estes mesmos flavonoides estão presentes na Carapa guianensis e também na Uncaria guianensis, sendo necessário saber a quantidade exata dessa substância, visto que neste estudo não houve efeito fungicida. No trabalho de Nunez (2013) foi possível observar que a Carapa guianensis possui fungos endofíticos, os quais podem ou não afetar no desempenho da mesma nos testes previamente mencionados, sendo necessário mais pesquisas para se obter a importância desses componentes presentes nela.

A ação da Uncaria guianensis sobre os fungos testados foi nula, entretanto existem relatos de eficácia contra cepas de Candida albicans assim como Microsporum canis (MORAES et al., 2011), o que se leva a acreditar que a mesma tenha sofrido alguma alteração química no preparo do extrato ou a dose e concentração utilizada foi muito baixa ou que realmente não tenha atividade contra o agente da Malassezia pachydermatis diretamente.

\section{CONCLUSÃO}

Os resultados obtidos nesse estudo indicam que a Carapa guianensis e a Uncaria guianensis não possuem atividade antifúngica significante perante a Malassezia pachydermatis isolada de primatas não humanos na concentração de $100 \mathrm{mg} / \mathrm{mL}$. É necessário, salientar que ambas as plantas possuem diversos efeitos terapêuticos, logo, podem possuir ação antifúngica, sendo necessária a realização de mais testes envolvendo os extratos destas plantas em concentrações mais altas.

\section{REFERÊNCIAS}

ALBANO, A.P.N. Fungos e micoses em animais silvestres recebidos por Centros de Triagem, Pelotas, 2009. 83 f. Dissertação (Mestrado em Veterinária) Universidade Federal de Pelotas, Pelotas, 2009.Disponível em: http://guaiaca.ufpel.edu.br/handle/123456789/2537 
AMORIM, S.L. Ação antiparasitária de plantas medicinais da Amazônia Ocidental sobre nematódeos gastrintestinais de ovinos: Prospecção Fitoquímica, Potencial Antihelmíntico e Análise Toxicológica. 2016. 109 f. Tese (Doutorado em Medicina Veterinária), Universidade Federal de Campina Grande, Campina Grande, 2016. Disponível

em: http://www.cstrold.sti.ufcg.edu.br/ppgmv/dissertacoes/teses/tese_2016/13_tese_sara_lu cena_de_amorim.pdf

CABAÑES, F.J.; VEGA, S.; CASTELLÁ, G. Malassezia cuniculi sp. nov, A novel yeast species isolated from rabbit skin. Medical Mycology, v. 49, p. 44-48, 2011. DOI: https://doi.org/10.3109/13693786.2010.493562

CARDOSO, R. L.; MABONI, F.; MACHADO, G.; ALVES, S.H.; VARGAS, A.C. Antimicrobial activity of propolis extract against staphylococcus coagulase positive and Malassezia pachydermatis of canine otitis. Veterinary Microbiology, v.142, p.432-434, 2010. DOI: https://doi.org/10.1016/j.vetmic.2009.09.070

CARTAXO, S.L.; SOUZA, M.M.A.; ALBUQUERQUE, U.P. Medicinal plants with bioprospecting potential used in semi-arid northeastern Brazil. Journal of Ethnopharmacology. v.131, n.2, p.326-342, 2010. DOI: https://doi.org/10.1016/ j.jep.2010.07.003.

CASTEJON, F.V. Taninos e saponinas. 2011. 29 f. Seminários Aplicados do Programa de Pós-Graduação, Universidade Federal de Goiás, Goiânia, 2011.Disponível em: https://files.cercomp.ufg.br/weby/up/67/o/semi2011_Fernanda_Castejon_1c.pdf

FARIAS, M.P.O.; SOUSA, D.P.; ARRUDA, A.C.; ARRUDA, M.S.P.; WANDERLEY, A.G., ALVES, L.C.; FAUSTINO, M.A.G. Eficácia in vitro do óleo da Carapa guianensis Aubl. (andiroba) no controle de Boophilus microplus (Acari: Ixodidae). Revista Brasileira de Plantas Medicinais v. 9, p. 68-71, 2007. Disponível em https:// www.scielo.br/ scielo.php?script=sci_nlinks\&ref=000065\&pid=S01020935200900040001500008\&lng=pt

FERRAZ, I.D.K.; CAMARGO, J.L.C.; SAMPAIO, P.T.B. Sementes e plântulas de andiroba (Carapa guianensis Aubl. e Carapa procera D.C.): aspectos botânicos, ecológicos e tecnológicos. Acta Amazônica, v. 32, n 4, p. 647-661, 2002. DOI: https://doi.org/1809-43922002324661.

FOY, D.S.; TREPANIER, L.A. Antifungal Treatment of Small Animal Veterinary Patients. Veterinary Clinical Small Animals, v. 40, n. 6, p. 1171-1188, 2010. DOI: https://doi.org/10.1016/j.cvsm.2010.07.006.

GUILLOT, J.; GUÉHO, E.; MIALOT, M.; CHEREMETTE, R. Importance des levures du genre Malassezia en dermatologie vétérinaire. Le Point 
Véetérinaire, v. 29, p. 21-31, 1998.disponível em http://alizarine.vetagro-sup.fr/dpchat/parasites/malmalass.html

HEIMERDINGER, A.; OLIVO, Z.; SCARAVELLI, L.F.B.; SKONIESKI, F.R.; AMBOS, J.F.; CHARÃO, P.S. Extrato alcoólico de capim cidreira (Cymbopogon citratus) no controle do Boophilus microplus em bovinos. Revista Brasileira de Parasitologia Veterinária, v. $15, \quad$ n. $1, \quad$ p. 37-9, 2006 . DOI: https:// www.researchgate.net/ publication / 283500373

HEITZMAN, M.E.; HEITZMAN, M.E.; NETO, C.C.; WINIARZ, E.; VAISBERG, A.J.; HAMMOND, G.B. Ethnobotany, phytochemistry and pharmacology of Uncaria $\begin{array}{llllll}\text { (Rubiaceae). } & \text { Phytochemistry, } & \text { v. 66, }\end{array}$ DOI: https://doi.org/10.1016/j.phytochem.2004.10.022.

HENRIQUES, M.D.; PENIDO, C. The therapeutic properties of Carapa guianensis. Current Pharmaceutical Design, v. 20 n. 6, p. 850-6, 2014. DOI: https://doi.org/10.2174/13816128113199990048.

ILKIT, M.; DURDU, M. Tinea pedis: the etiology and global epidemiology of a common fungal infection. Critical Reviews in Microbiology, v. 41, n. 3, p.374-88, 2015. DOI: https://doi.org/10.3109 / 1040841X.2013.856853.

KLAUCK, V.; PAZINATO, R.; RADAVELLI, W.M.; VOLPATO, A.; STEFANI, L.M. et al. In vitro repellent effect of tea tree (Melaleuca alternifolia) and andiroba (Carapa guianensis) oils on Haemotobia irritans and Chrysomya megacephalaflies. Tropical Biomedicine, v. 32, n. 1, p. 160-6, 2015. Disponível em https://www.researchgate.net/publication/274086616

LÓPEZ, R.J. Dermatitis canina por Malassezia. REDVET - Revista Electrónica de Veterinaria, v. 9, n. 5, p. 12-14, 2008. Disponível em www.revista.inf.br - www.editorafaef.com.br - www.faef.br.

MACHADO, R.M.A.; SOUZA, C.L.M.; FREIRE, M.G.M.; DIAS, V.M.; SILVA, L.B. Avaliação de óleos essenciais sobre o crescimento in vitro do fungo Colletotrichum gloeosporioides. Perspectiva Online, v.8, n.3, p.64-75, 2013.DOI: https: // doi.org/ 10.25242/ 8868382013147

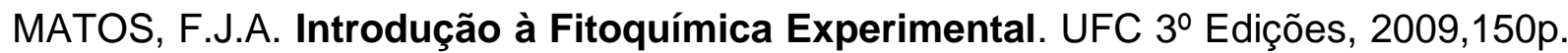
Disponível em: http://www.editora.ufc.br/ catalogo/ 21-ciencias-puras/ 346-introducao-afitoquimica-experimental-3-edicao

MELCHERT, A.; JEFERY, A.B.S.; GIUFFRIDA, R. Avaliações Citológicas Em Otites Caninas por Malassezia spp.: Estudo Retrospectivo - Colloquium Agrariae, v. 7, n. 2 p. 27-34, 2012. Disponível em: <http://revistas.unoeste.br/index.php/ca/article/view/646>. 
MIRANDA, E.M.; SOUSA, J.A.; PEREIRA, R.C.A. Caracterização e avaliação de populações nativas de unhade-gato [Uncaria tomentosa (Willd.) DC. e U. Guianensis (Aubl.) Gmel.] no vale do rio Juruá-AC. Revista Brasileira de Plantas Medicinais, v. 5, p. 41-46, 2003. Disponível em: <https://ainfo.cnptia.embrapa.br/digital/bitstream/item/111466/1/11481.pdf>.

MORAES, R.C. Investigação in vitro da atividade antifúngica de Uncaria tomentosa (WILLD) D.C. frente a leveduras patogênicas. 2011. 30 f. Faculdade de Farmácia, Universidade Federal do Rio Grande do Sul, Porto Alegre, 2011.Disponível em:

https://www.lume.ufrgs.br/bitstream/handle/10183/134858/000866456.pdf?sequence=1

NASCIMENTO, S.M.C.; CARVALHO, E.A.; WARWICK, D.R.N.; PALHETA, J.G.; SANTOS, T.P.F. Inibição do crescimento micelial de Thielaviopsis paradoxa por óleos vegetais. In: Encontro Amazônico de Agrárias, 5., 2013. Anais... Belém, PA, p.1-5, 2013. Disponível em: <https://www.embrapa.br/busca-de-publicacoes//publicacao/979377/inibicao>.

National Committee for Clinical Laboratory (NCCLS). Standards Performance Standards for Antimicrobial Disk Susceptibility Tests. NCCLS document M2-A8, Pennsylvania, USA: Edition Wayne, 2003, 58p. Disponível em: http:// www.anvisa.gov.br/ service saude/manuais/clsi/clsi_OPASM2-A8.pdf

NEVES, O.S.C.; BENEDITO, D.S.; MACHADO, R.V.; CARVALHO, J.G. Crescimento, produção de matéria seca e acúmulo de $\mathrm{N}, \mathrm{K}, \mathrm{Ca}, \mathrm{Mg}$, e $\mathrm{S}$ na parte aérea de mudas de andiroba (Carapa guianensis Aubl.) cultivadas em solo de várzea, em função de diferentes doses de fósforo. Revista Árvore, v. 28, n. 3, p. 343-9, 2004. DOI: https://doi.org/10.1590/S0100-67622004000300004.

NOGUEIRA, M.A.A.; SANTOS, A.S.; LEAL, C.A.S.; LIMA, D.C.V.; OLIVEIRA, E.A.A. et al. Diagnóstico das Dermatites Fúngicas em Cães e Gatos. In:> Jornada de Ensino, Pesquisa e Extensão - JEPEX/UFRPE, 13., 2013, Anais... UFRPE: Recife, p.1-3, 2013. Disponível em : http://www.eventosufrpe.com.br/2013/cd/listaresumos.htm

NUNEZ, C.V.; JACYNTHO, P.G.F. Isolamento e Identificação de fungos endofíticos de Carapa guianensis e Duroia macrophylla. In: Congresso de Iniciação Científica PIBIC/CNPq - PAIC/FAPEAM, 2., 2013. Anais... Manaus, p.1-4, 2013.Disponível em : http://portal.inpa.gov.br/arquivos/relatorios/TCG/INPA_TCG_2013_Anual_ANEXOS.pdf

PINTO, T.J.A; KANEKO T.M; Controle Biológico de Qualidade de Produtos Farmacêuticos, Correlatos e Cosméticos. 2. ed. São Paulo: Atheneu Editora, 2003. 325 p. Disponível em: https://www.scielo.br/scielo.php?script=sci_arttext\&pid=S1516$93322005000200018 \&$ Ing $=$ en\&nrm $=$ iso

POLLITO, P.A.Z. Dendrologia, anatomia do lenho e status de conservação das espécies lenhosas dos gêneros Cinchona, Croton e Uncaria no estado do Acre, Brasil. 2004. 181 f. Tese (Doutorado) - Escola Superior de Agricultura Luiz de Queiroz, 
Universidade de São Paulo, São Paulo, 2004. Disponível em: https://bdtd.ibict.br/vufind/Record/USP_eb2ee0438aadb4bd4ed7f1d5315a95e4

REINHARD, K.H. Uncaria Guianensis (Willd.) D.C.: Cat's Claw, Uña de Gato, or Saventaro. The Journal of Alternative Complementary Medicine, v. 5, p. 143-151,1999. DOI: 10.1089 / acm.1999.5.143.

REZENDE, C.; BORSARI, G.P.; SILVA, A.C.F.; CAVALCANTI, F.R. Estudo epidemiológico das dermatofitoses em instituições públicas da cidade de Barretos, São Paulo, Brasil. Revista Brasileira de Análise Clínica, v. 40, p. 13-16, 2008. Disponível em: <https://pesquisa.bvsalud.org/portal/resource/pt/lil-510669>.

SANDOVAL, D.M.R. Caracterización de especies de malassezia como microbiota comensal en la piel de equinos. 2014. $42 \mathrm{f}$. Monografia - Pontificia Universidad Javeriana Facultad de Ciencias Carrera de Bacteriología, Bogotá, 2014.Disponível em: https://repository.javeriana.edu.co/handle/10554/16674

SILVA, C.E.; SANTOS, O.J.; RIBAS-FILHO, J.M.; TABUSHI, F.I.; KUME, H. et al. Effect of Carapa guianensis Aublet (Andiroba) and Orbignya phalerata (Babassu) in colonic healing in rats. Revista do Colégio Brasileiro de Cirurgiões, v. 2, n. 6, p. 399-406, 2015. DOI: https://doi.org/10.1590/0100-69912015006009.

SIMÕES, C.M.O.; SCHENKEL, E.P.; MELLO, J.C.P.; MENTZ, L.A.; PETROVICK, P.R. Farmacognosia: do produto natural ao medicamento. Porto Alegre: Artmed, 2017. 486 p. Disponível em: https://www.scielo.br/pdf/rbfar/v12n1/a05v12n1.pdf

SOJAKOVA, M. et al. Fluconazole and Itraconazole susceptibility of vaginal yeast isolates from Slovakia. Mycophatologia, v. 157, n.2, p. 163-9, 2004.Disponível em: https://link.springer.com/article/10.1023/B:MYCO.0000020594.35357.b0

TRABULSI, L.R.; ALTERHUM, F. Microbiologia. 8르 ed. Rio de Janeiro: Atheneu, p. 55$509,2008$.

VITAL, T.M.; REIS, C.; GARCÍA-ZAPATA, M.T.A.; CUNHA, L.C. Estudo comparativo de duas técnicas farmacopéicas de avaliação da atividade antimicrobiana dos fármacos: nistatina, eritromicina, neomicina e gentamicina. Revista Brasileira de Ciências Farmacêuticas/ Brazilian Journal of Pharmaceutical Sciences, v. 40, n. 2, p. 219227, 2004. DOI: https://doi.org/10.1590/S1516-93322004000200011. 